\title{
Questionários dos Valores Básicos - Diagnóstico (QVB-D): Evidências de Validade de Construto
}

\author{
Basic Values Survey - Diagnosis (BVS-D): Evidence of Construct Validity
}

\author{
Luana Elayne Cunha de Souza ${ }^{*}, a$, Valdiney Veloso Gouveia ${ }^{a}$, Tiago Jessé Souza de Lima ${ }^{a}$ \\ $\&$ Walberto Silva dos Santos ${ }^{b}$ \\ ${ }^{a}$ Universidade Federal da Paraíba, João Pessoa, PB, Brasil \\ $\&{ }^{b}$ Universidade Federal do Ceará, Fortaleza, CE, Brasil
}

\begin{abstract}
Resumo
Este estudo teve como objetivo construir o Questionário dos Valores Básicos, versão diagnóstico (QVB-D), reunindo evidências de sua validade de construto (validades fatorial e convergente e consistência interna). No caso, teve-se em conta a teoria funcionalista dos valores humanos, identificando suas subfunções e três valores para representar cada uma. Participaram 210 estudantes do ensino médio e universitários de João Pessoa (PB), com idade média de 18,3 anos $(D P=2,94)$, a maioria do sexo feminino $(61,4 \%)$. Estes responderam o QVB-D e perguntas demográficas. Para conhecer evidências de validade fatorial do instrumento, foram realizadas análises fatoriais confirmatórias, ademais para avaliar a consistência interna três indicadores foram utilizados. Os resultados apoiaram a adequação de sua versão com 54 itens, mostrando evidências de validade de construto. Conclui-se que este é um instrumento psicometricamente adequado, podendo ser utilizado em pesquisas futuras e na prática profissional do psicólogo.

Palavras-chave: Valores, funções, medida, validade, diagnóstico.
\end{abstract}

\begin{abstract}
This study aimed to develop the Basic Values Survey, diagnosis version (BVS-D), gathering evidence of its construct validity (factorial and convergent validities and reliability). The functional theory of human values was taken into account, identifying its subfunctions and three values to represent each one. Participants were 210 school and university students in João Pessoa (PB), at a mean age of 18.3 years $(S D=2.94)$, mostly female $(61.4 \%)$. They answered the BVS-D and demographic questions. In order to know factorial validity evidence of the instrument, confirmatory factor analyses were performed, and to assess the internal consistency, three indicators were used. Results supported the appropriateness of its version with 54 items, showing evidence of construct validity. In conclusion, this instrument showed appropriate psychometric parameters, which justifies its use in further studies and professional practice of psychologists.

Keywords: Values, functions, measure, validity, diagnosis.
\end{abstract}

Uma das características que tornam os valores tão importantes é o seu impacto, direto ou indireto, sobre o comportamento (Roccas \& Sagiv, 2010), pois a forma natural das pessoas buscarem valores é se comportarem de maneira que os expressem ou os promovam (Bardi \& Schwartz, 2003). Deste modo, os valores são empregados para explicar uma variedade de comportamentos, tais como: preocupação com o meio ambiente (Groot \& Steg, 2008; Milfont, Sibley, \& Duckitt, 2010), condutas antissociais (Santos, 2008), religiosidade (Santos, 2008; Schwartz \&

* Endereço para correspondência: Universidade Federal da Paraíba, Programa de Pós-Graduação em Psicologia Social, João Pessoa, PB, Brasil 58051-900. E-mail: luana_elayne@hotmail.com.

Este artigo contou com apoio do Conselho Nacional de Desenvolvimento Científico e Tecnológico (CNPq) por meio de bolsa de mestrado concedida ao primeiro autor.
Huismans, 1995), preconceito (Vasconcelos, Gouveia, Souza, Sousa, \& Jesus, 2004), comportamento sexual de risco (Goodwin et al., 2002; Tamayo, Lima, Marques, \& Martins, 2001), comprometimento organizacional (Tamayo, 2005), orientação política e voto (Caprara, Schwartz, Capanna, Vecchione, \& Barbaranelli, 2006; Devos, Spini, \& Schwartz, 2002).

Neste sentido, parece evidente a contribuição que o estudo dos valores humanos oferece nos mais diversos âmbitos de atuação do psicólogo. Por exemplo, na psicoterapia, sua importância já vem sendo reconhecida há décadas (Kelly, 1990). Nas palavras de Rokeach (1973) "valores, atitudes e comportamentos podem sofrer mudanças duradouras quando as pessoas se tornam conscientes de certas contradições dentro de si” (p. 330). No contexto organizacional, Tamayo (2005) defende a importância de considerar os valores dos funcionários por parte da orga- 
Souza, L. E. C., Gouveia, V. V., Lima, T. J. S. \& Santos, W. S. (2015). Questionários dos Valores Básicos - Diagnóstico (QVB-D): Evidências de Validade de Construto.

nização, para o estabelecimento de vínculos saudáveis e a promoção do bem-estar.

Não obstante, para que a pesquisa sobre o papel dos valores nos contextos de atuação do psicólogo progrida, a mensuração dos valores deve ser assegurada de maneira válida e precisa, contudo essa área ainda apresenta limitações, como as comentadas a seguir. A medição dos valores humanos transcorreu de forma controversa e irregular até os anos 1970, de certa forma resultante da fragmentação teórica e diversidade conceitual que tornaram confusa sua operacionalização (Braithwaite \& Scott, 1991). Entretanto, em anos mais recentes parecem resolvidos esses problemas, ao menos no que diz respeito às formulações teóricas (e.g., Gouveia, 2003; Inglehart, 1977; Schwartz, 1992). Porém, apesar disso, alguns problemas são evidentes nos instrumentos que se propõem a medir os valores, sobretudo no que diz respeito aos seus indicadores de consistência interna (Gouveia, 2013). Por outro lado, considerando a importância e centralidade deste construto no sistema cognitivo (Maio, 2010), parece primordial empreender esforços para superar esta limitação.

Mesmo sendo evidente na literatura a importância dos valores humanos, pouco tem sido efetivamente feito para desenvolver medidas mais voltadas para a prática em Psicologia que possam, por exemplo, ser utilizadas como medidas de diagnóstico dos valores pessoais de maneira a identificar quais a prioridades axiológicas do paciente em processo de psicoterapia, auxiliar na seleção de funcionários para as empresas, entre outras aplicações. Os instrumentos disponibilizados têm sido mais focados em pesquisas básicas, a exemplo do Rokeach Values Survey (Rokeach, 1973), Schwartz Values Survey (Schwartz, 1992) e o Questionário dos Valores Básicos (Gouveia, 2003). De fato, quando se tem em conta a lista de testes psicológicos aprovados até 2013 para uso pelo Sistema de Avaliação dos Testes Psicológicos (SATEPSI), do Conselho Federal de Psicologia, não se encontra qualquer medida a respeito. Diante deste contexto, pensou-se no presente estudo. Seu objetivo principal foi construir uma medida de valores humanos voltada para triagem e diagnóstico, reunindo evidências de validade de construto (validades fatorial e convergente e consistência interna).

\section{Conceituação e Medidas dos Valores Humanos}

Apesar de ter sido reconhecida a relevância dos valores para a atividade humana, quer no nível individual ou social de análise, o desenvolvimento dessa temática foi dificultado por problemas de definição e dúvidas acerca da própria viabilidade empírica do construto (Braithwaite \& Scott, 1991). A propósito, nos anos 1950 ainda não havia um referencial adequado para conceituar um valor, e, embora a noção deste ser um atributo absoluto de um objeto tenha sido rejeitada, ainda não havia consenso em relação a outros aspectos, como, por exemplo, a distinção entre valor como desejável ou desejado. No caso, foi somente a partir da definição de Kluckhohn (1951) que um consenso emergiu, concebendo os valores como "centrados na pessoa" e pertencentes ao "desejável".

Mesmo com algum avanço no nível conceitual, a convergência nas pesquisas empíricas sobre os valores esbarrava na falta de acordo quanto ao plano operacional. A partir da revisão ampla acerca das medidas de valores realizada por Braithwaite e Scott (1991), constata-se que os valores eram tratados como coisas diferentes, a exemplo de interesses (Allport, Vernon, \& Lindzey, 1960), filosofias de vida (Morris, 1956) ou comportamentos morais (Harding \& Phillips, 1986). Este era, então, o estado da arte que evidenciava certa falta de sinergia conceitual e operacional na esfera dos estudos sobre valores.

O trabalho de Rokeach $(1968,1973)$ foi decisivo para mudar o rumo dos estudos sobre os valores. Uma de suas contribuições destacáveis foi no plano conceitual, propondo uma definição que os diferenciavam de construtos correlatos (e.g., atitudes, traços de personalidade). Ademais, contribuiu para sua operacionalização, designando-os como modos de conduta ou estados finais de existência. A partir de suas contribuições, as pesquisas sobre os valores tiveram um rumo diferente, focando em abordagem mais empírica e menos filosófica. Estes aspectos se evidenciam na elaboração do Rokeach Values Survey, considerado o primeiro instrumento específico para medir os valores.

Os estudos sobre os valores não se encerraram com as contribuições de Milton Rokeach. Depois dele foram propostas teorias que são mais elaboradas, a exemplo daquela proposta por Shalom H. Schwartz, que integra manuais e capítulos de livro da Psicologia (Maio, 2010; Maio, Olson, Bernard, \& Luke, 2006; Smith \& Schwartz, 1997). Contudo, tiveram lugar outras teorias, inclusive prévia àquela comentada anteriormente, proposta por Inglehart (1977) e também mais recentemente (Gouveia, 2003; Gouveia, Milfont, \& Guerra, 2014). Porém, os problemas acerca das medidas seguiram existindo, as quais se limitaram ao uso em pesquisas, visando conhecer os correlatos dos valores. Resgatam-se alguns dos pontos críticos das medidas desses autores.

O Rokeach Values Survey tem sido criticado pelo uso de palavras ou frases muito curtas, o que geram múltiplas interpretações, distorcendo seus significados em culturas distintas (Gibbins \& Walker, 1993). Também tem sido objeto de restrição em decorrência de sua natureza ipsativa, proporcionando pouca informação e correlações baixas entre seus itens (Gorsuch, 1970). Por outro lado, a crítica principal feita ao instrumento de Inglehart (1977), o World Values Survey, diz respeito à organização de seus itens em dois polos presumivelmente opostos, isto é, materialismo e pós-materialismo, tornando-o inadequado em função dos dados produzidos não condizerem com a realidade, mostrando que as culturas podem ser ao mesmo tempo materialistas e pós-materialistas (Inglehart, Basañez, \& Moreno, 1998).

Com respeito à medida de Schwartz (1992; Schwartz Values Survey), existem vários aspectos que são criticados 
(Gouveia, Milfont, Fischer, \& Santos, 2008). Por exemplo, há uma ressalva quanto ao uso da classificação de sua lista de valores em instrumentais e terminais; também se critica o emprego de escala de resposta assimétrica, variando de -1 a 7 , que tem sido falha em identificar os valores supremos (Tamayo, 2007). Estes dois aspectos, provavelmente, refletem a influência que esse autor recebeu de Rokeach (1973). Porém, provavelmente a crítica mais contundente diga respeito à carência de um modelo teórico sólido que embase seu conjunto de tipos motivacionais, refletindo em números diferentes de itens para cada tipo (e.g., hedonismo abarca dois itens, enquanto universalismo reúne oito).

Finalmente, o modelo de Gouveia (2003) deu origem ao Questionário dos Valores Básicos, que também não está isento de limitações. Por exemplo, apesar de não ser uma característica restrita de sua medida (Schwartz, 2005), os coeficientes de consistência interna de seus fatores (suas subfunções) são comumente baixos, provavelmente em razão da própria natureza do construto avaliado, mas também do número reduzido de itens que os compõe, três para cada um (Pasquali, 2003). Destaca-se, ainda, que cada valor específico é representado por duas definições breves, que podem não retratar toda extensão da ideia do valor subjacente.

Em resumo, frente ao que foi exposto, considerando o histórico problemático da mensuração dos valores humanos e as críticas brevemente apresentadas de alguns dos principais instrumentos (Braithwaite \& Scott, 1991), incluindo a medida elaborada no contexto brasileiro (Gouveia, 2003), percebe-se que ainda há uma lacuna a ser preenchida. Concretamente, demanda-se elaborar uma medida que reúna melhores indicadores de validade e precisão, pautada em teoria sólida dos valores, favorecendo seu emprego nos contextos de triagem e diagnóstico psicológicos. Este aspecto favoreceu pensar na presente proposta, que visou oferecer uma medida pautada na teoria funcionalista dos valores (Gouveia, 2003, 2013; Gouveia et al., 2008; Gouveia et al., 2010). Neste sentido, carece descrevê-la brevemente.

\section{Teoria Funcionalista dos Valores Humanos}

Esta teoria foi elaborada inicialmente nos anos 1990 (Gouveia, 1998), tendo sido aprimorada nos últimos anos (Gouveia, 2003, 2013; Gouveia et al., 2014; Gouveia, Milfont, Fischer, \& Coelho, 2009; Gouveia et al., 2008; Gouveia et al., 2010). Ela não pode ser considerada como uma contraposição a modelos prévios que consideram a natureza motivacional dos valores humanos (e.g., Inglehart, 1977; Rokeach, 1973; Schwartz, 1992). Trata-se, contrariamente, de uma proposta integradora, embora parcimoniosa, contribuindo para o desenvolvimento teórico da área, que considera as seguintes características para a definição dos valores: (a) são conceitos ou categorias, (b) sobre estados desejáveis de existência, (c) que transcendem situações específicas, (d) assumem diferentes graus de importância, (e) guiam a seleção ou avaliação de comportamentos e eventos e (f) representam cognitivamente as necessidades humanas (Inglehart, 1977; Kluckhohn, 1951; Rokeach, 1973; Schwartz, 1992). Não obstante, seu foco principal são as funções dos valores, tratadas a seguir.

Funções e Subfunções dos Valores. Gouveia (1998, 2003; Gouveia et al., 2009; Gouveia et al., 2008; Gouveia et al., 2010) tem identificado na literatura duas funções consensuais: (a) guiam as ações do homem (tipo de orientação; Rokeach, 1973; Schwartz, 1992) e (b) expressam suas necessidades (tipo de motivador; Inglehart, 1977; Maslow, 1954). Estas funções são detalhadas a seguir.

A função de guiar os comportamentos humanos é identificada pela dimensão tipo de orientação, que pode ser social, central e pessoal. As pessoas guiadas por valores sociais são centradas na sociedade, com ênfase nas relações interpessoais; por outro lado, aquelas guiadas por valores pessoais são egocêntricas, possuindo um foco intrapessoal. Portanto, as pessoas tendem a enfatizar o grupo (valores sociais) ou elas mesmas (valores pessoais) como a unidade principal de sobrevivência. Entretanto, existe um terceiro grupo de valores que não são completa ou exclusivamente social ou pessoal (Gouveia, 1998, 2003; Schwartz \& Bilsky, 1987). Schwartz (1992) os denomina como valores mistos ou tipos motivacionais mistos. Porém, ele não justifica a razão de tais valores aparecerem entre os sociais e pessoais. A presente teoria propõe que tais valores se situam entre os sociais e pessoais porque compreendem sua base organizadora, isto é, os demais valores o têm como referência, expressando as necessidades mais básicas (e.g., comer, beber) e as de ordem superior (e.g., beleza, cognição). Por isso, este terceiro grupo de valores é referido como central.

A função de expressar as necessidades humanas é representada pela dimensão funcional tipo de motivador, da qual derivam os valores materialistas (pragmáticos) ou idealistas (humanitários; Inglehart, 1977). Os valores materialistas estão relacionados com ideias práticas, sugerindo uma orientação para metas específicas e regras normativas. Indivíduos guiados por estes valores tendem a pensar em condições de sobrevivência mais biológicas, priorizando sua própria existência e as condições nas quais esta é assegurada. Por outro lado, os valores idealistas expressam uma orientação universal, baseada em ideias e princípios mais abstratos. Comparados com os valores materialistas, os idealistas não são dirigidos a metas concretas e, geralmente, são não específicos. Dar importância a valores idealistas é coerente com um espírito inovador, havendo menos dependência de bens materiais.

Em síntese, as duas funções valorativas formam dois eixos a partir dos quais os valores são organizados estruturalmente: tipo de orientação (valores sociais, centrais e pessoais) e tipo motivador (valores materialistas e idealistas). A combinação destes eixos permite derivar seis subfunções específicas dos valores, descritas na Figura 1. 
Souza, L. E. C., Gouveia, V. V., Lima, T. J. S. \& Santos, W. S. (2015). Questionários dos Valores Básicos - Diagnóstico (QVB-D): Evidências de Validade de Construto.

\begin{tabular}{|c|c|c|c|c|}
\hline & & \multicolumn{3}{|c|}{ Valores como padrão-guia de comportamentos } \\
\hline & & $\begin{array}{l}\text { Metas pessoais (o } \\
\text { indivíduo por si } \\
\text { mesmo) }\end{array}$ & $\begin{array}{c}\text { Metas centrais (o } \\
\text { propósito geral da } \\
\text { vida) }\end{array}$ & $\begin{array}{c}\text { Metas sociais (o } \\
\text { indivíduo na } \\
\text { comunidade) }\end{array}$ \\
\hline 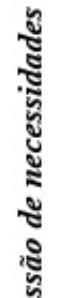 & $\begin{array}{l}\text { Necessidades } \\
\text { idealistas (a vida } \\
\text { como fonte de } \\
\text { oportunidades) }\end{array}$ & $\begin{array}{c}\text { Experimentação } \\
\text { Emoção } \\
\text { Prazer } \\
\text { Sexualidade }\end{array}$ & $\begin{array}{c}\text { Suprapessoal } \\
\text { Beleza } \\
\text { Conhecimento } \\
\text { Maturidade }\end{array}$ & $\begin{array}{l}\text { Interativa } \\
\text { Afetividade } \\
\text { Apoio social } \\
\text { Convivência }\end{array}$ \\
\hline 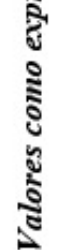 & $\begin{array}{c}\text { Necessidades } \\
\text { materialistas (a } \\
\text { vida como fonte } \\
\text { de ameaças) }\end{array}$ & $\begin{array}{l}\text { Realização } \\
\text { Êxito } \\
\text { Poder } \\
\text { Prestígio }\end{array}$ & $\begin{array}{c}\text { Existência } \\
\text { Estabilidade } \\
\text { Saúde } \\
\text { Sobrevivência }\end{array}$ & $\begin{array}{c}\text { Normativa } \\
\text { Obediência } \\
\text { Religiosidade } \\
\text { Tradição }\end{array}$ \\
\hline
\end{tabular}

Figura 1. Funções, subfunções e valores específicos.

Subfunção experimentação. A necessidade fisiológica de satisfação, em sentido amplo (princípio do prazer), é representada por valores desta subfunção. Ela é menos pragmática na busca de alcançar status social ou assegurar harmonia e segurança sociais; seus valores contribuem para a promoção de mudanças na estrutura de organizações sociais, sendo mais endossados por jovens. São indicadores desta subfunção os valores emoção, prazer e sexualidade.

Subfunção realização. As necessidades de autoestima são representadas por valores desta subfunção. Seus valores se originam de um princípio pessoal para guiar a vida dos indivíduos, enquanto focaliza realizações materiais; podem ser uma exigência para interações sociais prósperas e o funcionamento institucional. Tais valores são mais tipicamente apreciados por jovens adultos, em fase produtiva, ou indivíduos educados em contextos disciplinares e formais. São exemplos de valores desta subfunção os seguintes: êxito, poder e prestígio.

Subfunção suprapessoal. Esta subfunção tem uma orientação central, representando seus valores as necessidades estéticas e de cognição, bem como a necessidade superior de autorrealização. Eles podem ser concebidos como idealistas, indicando a importância de ideias abstratas, com menor ênfase em coisas concretas e materiais. A pessoa que endossa uma orientação central e um motivador idealista costuma pensar de forma mais geral e ampla, tomando decisões e se comportando a partir de critérios universais. Os três valores indicadores desta podem ser beleza, conhecimento e maturidade.

Subfunção existência. Representa as necessidades fisiológicas básicas (e.g., comer, beber, dormir) e a necessidade de segurança. O propósito principal de seus valores é garantir as condições básicas para a sobrevivência bio- lógica e psicológica do indivíduo. Os valores de existência são endossados por indivíduos em contextos de escassez econômica, ou por aqueles que foram socializados em tais ambientes. Três dos valores que podem representar esta subfunção são estabilidade pessoal, saúde e sobrevivência.

Subfunção interativa. A experiência afetiva entre indivíduos são ressaltados por seus valores, representando as necessidades de pertencimento, amor e afiliação, reunindo valores essenciais para estabelecer, regular e manter as relações interpessoais. Contatos sociais são uma meta em si, enfatizando atributos mais afetivos e abstratos. As pessoas que os adotam são frequentemente mais jovens e orientadas para relações íntimas estáveis. Três valores indicadores desta subfunção são afetividade, apoio social e convivência.

Subfunção normativa. Reflete a importância de preservar a cultura e as normas convencionais. Priorizar valores normativos evidencia uma orientação vertical, na qual a obediência à autoridade é importante. Pessoas mais velhas são mais prováveis de serem guiadas por tais valores, seguindo normas convencionais e tendo menos comportamentos anticonvencionais. Três valores que a representam são obediência, religiosidade e tradição.

Existem instrumentos pautados na teoria funcionalista que permitem apreender as subfunções valorativas em pessoas da população geral (QVB; Gouveia, 2003) e crianças (QVB-I; Gouveia, Milfont, Soares, Andrade, \& Leite, 2011), tendo a vantagem de reunirem apenas 18 itens, o que facilita a coleta de dados. Entretanto, reconhece-se a necessidade de um instrumento mais amplo, que reúna indicadores de validade e precisão mais robustos, necessários para endossar decisões na prática profissional. Nesta direção, decidiu-se elaborar um instrumento que possa ser 
utilizado em âmbitos diferentes de atuação psicológica (e.g., clínico, organizacional, escolar, jurídico), servindo como ferramenta ou recurso de triagem e diagnósticos de prioridades axiológicas.

Em resumo, decidiu-se propor uma medida de valores, pautada na teoria funcionalista, considerando número maior de itens, buscando reunir evidências de sua validade fatorial e convergente e consistência interna. Este instrumento será denominado como QVB-D (Questionários dos Valores Básicos - Diagnóstico).

\section{Método}

\section{Participantes}

Participaram deste estudo 210 estudantes do ensino médio de uma escola privada $(39,5 \%)$ e universitários da cidade de João Pessoa (PB). Estes tinham idades variando de 14 a 28 anos $(M=18,3 ; D P=2,94)$, sendo a maioria do sexo feminino $(61,4 \%)$ e católica $(55,8 \%)$.

\section{Instrumento}

Inicialmente, buscou-se contar com um conjunto de itens que representassem as seis subfunções antes descritas. Partiu-se do banco de 200 itens de Gouveia (1998), selecionando aqueles como correlações item-total iguais ou superiores a 0,30 , resultando em 178 itens. Estes foram traduzidos do espanhol para o português por um pesquisador bilíngue. O passo seguinte, almejando que todas as subfunções fossem representadas pela mesma quantidade de itens, decidiu-se elaborar mais itens para as subfunções com menos de 30 itens e excluir outros para aquelas que excediam este número. Desta forma, chegou-se a um instrumento com 180 itens igualmente distribuídos nas seis subfunções. Em seguida, procedeu-se a uma análise de juízes para realizar uma segunda seleção destes itens. No caso, contou-se com a participação de dez professores doutores, especialistas em valores, visando julgar a adequação de cada item para representar uma única subfunção. Utilizando como critério ao menos $70 \%$ de concordância entre os avaliadores, foram selecionados 127 itens, distribuídos como seguem: experimentação $(n=24)$, realização $(n=$ 27), existência $(n=21)$, suprapessoal $(n=14)$, interativa $(n=19)$ e normativa $(n=22)$.

Selecionados os itens para o instrumento novo, restava ainda mais um procedimento antes da coleta de dados: validação semântica. Esta contou com a participação de 12 estudantes do primeiro ano do ensino médio (extrato mais baixo da população alvo, isto é, estudantes de ensino médio e universitários) de uma escola particular. Nesta oportunidade, procurou-se verificar se eram compreensíveis as instruções sobre como responder a escala de resposta e os itens. Verificado que não ocorreram questionamentos, manteve-se esta versão proposta que é descrita a seguir; esta foi respondida juntamente com três perguntas demográficas (idade, religião e sexo).

A versão experimental do Questionário dos Valores Básicos - Diagnóstico (QVB-D) foi composta por 127 itens, distribuídos entre as seis subfunções valorativas, conforme previamente se indicou. Os itens pertencentes a cada subfunção representam os três valores humanos listados na Figura 1 (e.g., a subfunção experimentação foi representada pelos valores de emoção, prazer e sexualidade). Diferentemente do QVB em que cada item conta com duas descrições, no QVB-D cada item expressa uma única ideia (e.g. Buscar novas experiências). Empregouse escala de sete pontos para respondê-los, variando de 1 (Totalmente não importante) a 7 (Extremamente importante), de forma a expressar o grau de importância de cada valor como um princípio-guia para a vida da pessoa. Para avaliar quais as prioridades valorativas de cada indivíduo, deve-se criar um escore médio por subfunção e analisar quais subfunções são priorizadas.

\section{Procedimento}

Os instrumentos foram aplicados de forma coletiva em sala de aula, porém respondidos individualmente; bastava aos participantes seguirem as orientações dadas por escrito no próprio questionário. Em um primeiro momento, foram passados oralmente os esclarecimentos no que se referiu ao anonimato e sigilo de sua participação, bem como quanto às diretrizes éticas que regem a pesquisa com seres humanos. Nesta oportunidade, procurou-se que sua participação fosse consentida, devendo, assim, assinar o termo de consentimento livre e esclarecido. No caso de pessoas com menos de 18 anos, o diretor do estabelecimento educacional ou professor assinou termo de responsabilidade. O projeto foi aprovado pelo Comitê de Ética em Pesquisa com Seres Humanos, do Hospital Universitário Lauro Wanderley, da Universidade Federal da Paraíba (Proc. CEP/HULW No 143/11). Os presentes levaram, em média, 25 minutos para concluírem sua participação.

\section{Análise dos Dados}

Com o PASW 18 foram calculadas estatísticas descritivas e consistência interna, isto é, alfa de Cronbach, considerando-se como adequado valor acima de 0,70 (Nunnally, 1991), além da homogeneidade (correlação média inter-itens, $r_{\text {m.i. }}$ ) de cada subfunção, assumindo como adequados valores iguais ou superiores a 0,20 (Clark \& Watson, 1995). Com o fim de conhecer evidências de validade fatorial do instrumento, o AMOS (versão 18) foi empregado para realizar análises fatoriais confirmatórias, tendo em conta a matriz de covariância e adotando o estimador ML (Máxima Verossimilhança).

Para conhecer o ajuste dos modelos, utilizaram-se os seguintes indicadores: a razão qui-quadrado para o grau de liberdade $\left(\chi^{2} / g l\right)$, cujos valores entre 2 e 3 indicam um ajuste aceitável, admitindo-se até 5; o Goodness-of-Fit Index (GFI), o Adjusted Goodness-of-Fit Index (AGFI) e o Comparative Fit Index (CFI), onde valores iguais ou superiores a 0,90 inicam ajuste aceitável, com preferência para valores acima de 0,95; e o Root-Mean-Square Error of Approximation (RMSEA), assumindo-se como ideal valor situado entre 0,05 e 0,08, aceitando-se até 0,10 (Byrne, 2010). Além disso, o Expected Cross-Validation Index (ECVI) foi utilizado para avaliar melhoras entre os mode- 
Souza, L. E. C., Gouveia, V. V., Lima, T. J. S. \& Santos, W. S. (2015). Questionários dos Valores Básicos - Diagnóstico (QVB-D): Evidências de Validade de Construto.

los concorrentes testados, sendo útil para a comparação de modelos não aninhados; valores mais baixos de ECVI refletem um modelo com melhor ajuste (Brown, 2006).

Por fim, reunindo evidências complementares de validade de construto, procurou-se calcular a variância média extraída (VME) e a confiabilidade composta (CC; Fornell \& Larcker, 1981; Hair, Anderson, Tatham, \& Black, 1998). Valores iguais ou superiores a 0,50 e 0,70, respectivamente, asseguram a adequação da medida. A VME é indicadora de validade convergente de cada fator, isto é, o quanto ele serve para explicar o conjunto de itens. Por sua vez, o CC é um indicador adicional de consistência interna que pode dirimir dúvida quanto ao alfa de Cronbach (Pasquali, 2003), uma vez que tem a vantagem de não pressupor que os itens sejam tau equivalentes, ou seja, tenham iguais pesos fatoriais; também não pressupõe que os erros de medida sejam independentes e não é influenciado pelo número de itens.

\section{Resultados}

Evidências de Validade Fatorial e Convergente

Foram realizadas análises fatoriais confirmatórias para cada subfunção valorativa separadamente. Optou-se por este procedimento de forma a garantir que cada subfunção apresentasse fortes indicadores de validade e precisão sem influência das demais subfunções. Com base nos resultados dessas análises, foram selecionados três itens para cada valor humano, os quais deveriam obedecer a dois critérios: (a) maior carga fatorial e (b) abrangência do conteúdo do valor humano representado. Em seguida, foram realizadas novas análises fatoriais confirmatórias com a mesma estrutura fatorial, mas apenas com os itens selecionados de cada subfunção. Os índices de ajuste para os modelos com todos os itens (M1) e aqueles com os itens selecionados (M2) são apresentados na Tabela 1.

Tabela 1

Indicadores de Ajuste dos Modelos e Consistência Interna por Subfunção Valorativa do QVB-D

\begin{tabular}{|c|c|c|c|c|c|c|c|c|c|}
\hline Modelo & $\chi^{2} / g l$ & GFI & AGFI & CFI & $\begin{array}{l}\text { RMSEA } \\
(\text { IC 90\%) }\end{array}$ & ECVI & $\alpha$ & $r_{\mathrm{m} . \mathrm{i}}$ & $\mathrm{CC}$ \\
\hline Experimentação M1 & 3,21 & 0,74 & 0,68 & 0,78 & $\begin{array}{c}0,103 \\
(0,095-0,111)\end{array}$ & 4,311 & - & - & - \\
\hline Experimentação M2 & 3,73 & 0,91 & 0,83 & 0,89 & $\begin{array}{c}0,114 \\
(0,090-0,140)\end{array}$ & 0,629 & 0,79 & 0,29 & 0,61 \\
\hline Realização M1 & 2,22 & 0,80 & 0,77 & 0,81 & $\begin{array}{c}0,076 \\
(0,069-0,084)\end{array}$ & 3,670 & - & - & - \\
\hline Realização M2 & 3,25 & 0,92 & 0,86 & 0,90 & $\begin{array}{c}0,104 \\
(0,079-0,130)\end{array}$ & 0,574 & 0,80 & 0,32 & 0,81 \\
\hline Suprapessoal M1 & 2,93 & 0,87 & 0,82 & 0,75 & $\begin{array}{c}0,096 \\
(0,081-0,111)\end{array}$ & 1,334 & - & - & - \\
\hline Suprapessoal M2 & 2,26 & 0,95 & 0,90 & 0,90 & $\begin{array}{c}0,078 \\
(0,050-0,105)\end{array}$ & 0,460 & 0,72 & 0,22 & 0,92 \\
\hline Existência M1 & 2,42 & 0,82 & 0,78 & 0,78 & $\begin{array}{c}0,083 \\
(0,073-0,092)\end{array}$ & 2,587 & - & - & - \\
\hline Existência M2 & 2,18 & 0,95 & 0,91 & 0,92 & $\begin{array}{c}0,075 \\
(0,047-0,103)\end{array}$ & 0,452 & 0,77 & 0,28 & 0,67 \\
\hline Interativa M1 & 2,39 & 0,85 & 0,81 & 0,82 & $\begin{array}{c}0,081 \\
(0,071-0,092)\end{array}$ & 2,093 & - & - & - \\
\hline Interativa M2 & 4,15 & 0,90 & 0,82 & 0,87 & $\begin{array}{c}0,123 \\
(0,098-0,148)\end{array}$ & 0,677 & 0,84 & 0,37 & 0,73 \\
\hline Normativa M1 & 2,22 & 0,83 & 0,79 & 0,89 & $\begin{array}{c}0,076 \\
(0,067-0,086)\end{array}$ & 2,636 & - & - & - \\
\hline Normativa M2 & 2,82 & 0,94 & 0,88 & 0,95 & $\begin{array}{c}0,093 \\
(0,067-0,120)\end{array}$ & 0,524 & 0,83 & 0,35 & 0,84 \\
\hline
\end{tabular}

Nota . M1 = modelo com todos os itens da subfunção; M2 = modelo com os 9 itens selecionados por subfunção; $\alpha=$ Alfa de Cronbach, $r_{\mathrm{m} . \mathrm{i}}=$ Índice de Homogeneidade, $\mathrm{CC}=$ Confiabilidade Composta. 
Como pode ser observado na Tabela 1, os modelos com os itens selecionados são, em todas as subfunções, melhores do que aqueles com todos os itens. Isso se evidencia quando são observados os índices do ECVI que são menores para os modelos com os itens selecionados.
Ademais, todas as saturações (lambdas) dos itens destes modelos foram superiores a 0,30 , sendo estatisticamente diferentes de zero $(\lambda \neq 0 ; z>1,96, p<0,05)$, apresentando os fatores correlações positivas entre si $(\Phi$; valor Phi padronizado). Este resultado é resumido na Figura 2.
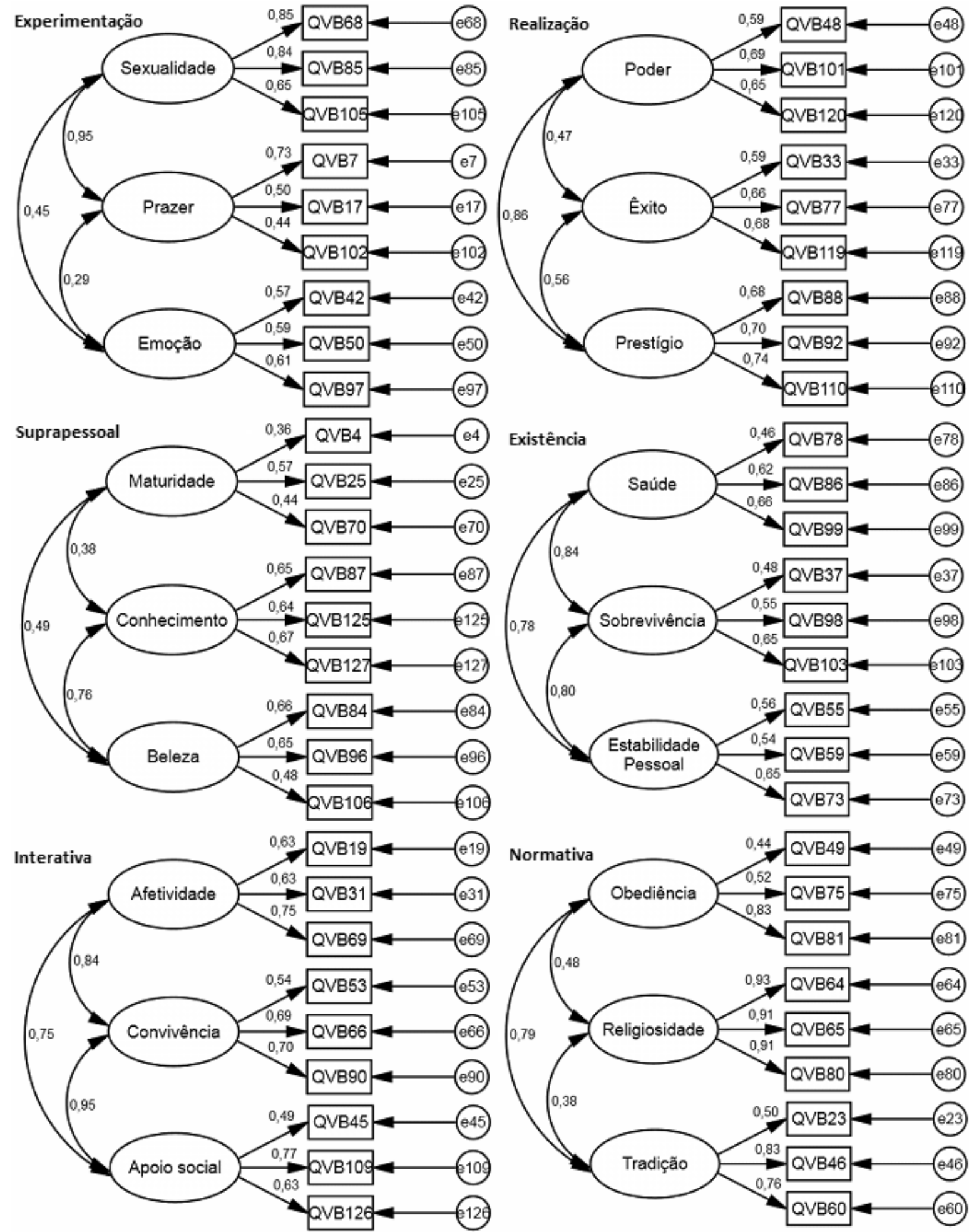

Figura 2. Estrutura fatorial das subfunções valorativas do QVB-D. 
Souza, L. E. C., Gouveia, V. V., Lima, T. J. S. \& Santos, W. S. (2015). Questionários dos Valores Básicos - Diagnóstico (QVB-D): Evidências de Validade de Construto.

Por fim, quanto a indicadores complementares de validade de construto desta medida, calculou-se a VME para cada fator, sendo os valores como seguem: 0,46 (suprapessoal), 0,53 (experimentação, existência e normativa), 0,56 (realização) e 0,61 (interativa).

\section{Evidências de Consistência Interna}

Complementando os resultados anteriormente descritos, reuniram-se evidências de consistência interna dos seis fatores ou subfunções valorativas. Estes achados também são descritos na Tabela 1. Como se pode observar na Tabela 1, todos os fatores apresentaram alfas de Cronbach acima do que é recomendado pela literatura, variando de 0,72 (suprapessoal) a 0,84 (interativa). Os índices de homogeneidade também se mostraram adequados para todos os fatores, variando de 0,22 (suprapessoal) a 0,37 (interativa). Por fim, reforçando este parâmetro, a confiabilidade composta dos fatores variou de 0,61 (experimentação) a 0,92 (suprapessoal).

\section{Discussão}

O objetivo principal deste estudo foi construir um instrumento novo para medir valores humanos (QVB-D), reunindo fortes evidências de seus parâmetros psicométricos de maneira a ser utilizado para fins de triagem e diagnóstico. Este, como se depreende dos resultados, ficou composto por 54 itens, sendo distribuídos equitativamente nas seis subfunções valorativas, cada uma composta por três valores humanos que concentraram três itens cada, em consonância com a Figura 1. Em geral, pode-se afirmar que este objetivo foi alcançado, sendo demonstradas evidências satisfatórias de validade fatorial e convergente e consistência interna (homogeneidade, alfa de Cronbach e Confiabilidade Composta). Neste ponto vale salientar que se buscou criar um instrumento psicometricamente adequado para o contexto da prática profissional dos psicólogos. Neste sentido, justifica-se a utilização de uma medida mais ampla, que busca abarcar toda a extensão do construto em questão.

Apesar do que anteriormente se comentou, antes de discutir os principais resultados deste estudo cabe reconhecer suas limitações potenciais. Neste caso, pode-se apontar como limitação, por exemplo, o fato de este estudo partir do mesmo banco de itens utilizado para construir o instrumento de Gouveia $(1998,2003)$. Como se trata de um trabalho de construção de instrumento, poderia se pensar na criação de itens novos. Contudo, pensa-se que este empreendimento seria desnecessário, já que tanto o QVB quanto o instrumento proposto se pautaram na mesma teoria dos valores. Destaca-se, ainda, que foi primado pela representatividade dos itens em relação ao construto, não sendo os marcadores valorativos (itens) o aspecto fundamental, mas sim as subfunções valorativas. Além disso, itens novos foram criados e incorporados ao instrumento, justamente para assegurar a adequação não só psicométrica, mas também para garantir que toda a exten- são de cada conteúdo do construto (subfunção valorativa) fosse abarcada.

Também é preciso frisar que este estudo se pautou em delineamento correlacional, empregando um instrumento autoaplicável, ou seja, constitui-se em uma medida de autorrelato, carecendo de um correspondente efetivamente comportamental. Portanto, estudos futuros podem buscar desenvolver outros tipos de medida para os valores humanos, como, por exemplo, focando em medidas implícitas. Entretanto, está em consonância com os instrumentos comumente empregados para a avaliação psicológica (Pasquali, 2003).

Gouveia e colegas (2009; Gouveia et al., 2008; Gouveia et al., 2010) afirmam que os valores são organizados de acordo com duas dimensões funcionais: tipo de orientação e tipo de motivador, cuja combinação origina seis subfunções valorativas, cada uma representada por três valores específicos. Esta estrutura fatorial foi testada para cada subfunção, tendo sido apresentados os resultados para dois modelos em cada subfunção valorativa: o primeiro com todos os itens da subfunção avaliada e o segundo correspondente aos itens selecionados para se chegar à estrutura fatorial almejada. Destaca-se que em todas as seis subfunções foram observados indicadores de ajuste melhores para o segundo modelo.

É importante observar que alguns indicadores de ajuste estiveram abaixo do que é sugerido na literatura. Por exemplo, o CFI não alcançou o 0,90 esperado em duas das subfunções (Byrne, 2010). Porém, Garson (2003) indica que mesmo um CFI abaixo deste valor pode ser admissível em um campo em que se costumam encontrar valores, por exemplo, de 0,70. Ressalta-se, neste âmbito, que a avaliação de ajuste de um modelo precisa ser feita em conjunto. A este respeito, os valores de $\chi^{2}$ e ECVI sempre menores para o modelo com os itens selecionados indicam seu melhor ajuste em comparação com aqueles com todos os itens. Adicionalmente, observaram-se evidências de validade convergente, uma vez que cinco das subfunções apresentaram valores de variância média extraída (VME) acima do recomendado pela literatura $(0,50$; Fornell \& Larcker, 1981), exceto a subfunção suprapessoal, com um valor um pouco inferior ao que é sugerido. Este achado, provavelmente, pode ser explicado em razão da natureza desse construto, que é mais comumente evidente em pessoas de maior idade (Gouveia, 2003, 2013); lembrando, os participantes foram jovens estudantes.

No que concerne à consistência interna das subfunções do QVB-D, todos os resultados se mostraram satisfatórios. Os valores de alfa de Cronbach foram acima do ponto de corte sugerido na literatura $(0,70$; Nunnally, 1991), assim como os coeficientes de homogeneidade $(0,20$; Clark \& Watson, 1995). Quanto à confiabilidade composta; embora sugerido idealmente valores na casa de 0,70 (Hair et al., 1998), admitem-se a partir de 0,60 (Fornell \& Larcker, 1981), suportando este parâmetro para todas as subfunções.

Em resumo, reuniram-se evidências empíricas acerca da adequação psicométrica do QVB-D. Estes achados 
são animadores, justificando seu emprego em pesquisas futuras, mas, também, nos contextos de prática profissional do psicólogo. A propósito, ressalta-se que este é o seu foco, sobretudo quando é possível considerar maior disponibilidade de tempo para seu uso, como evidenciado nos âmbitos clínico, escolar e organizacional, por exemplo. Buscar conhecer as prioridades valorativas de uma pessoa, em qualquer que seja o ambiente, é de extrema utilidade para o psicólogo. Em um contexto de seleção profissional, por exemplo, saber que valores são mais importantes como princípios-guia para um candidato a emprego, ajudará o psicólogo organizacional a julgar se tal candidato se encaixa no perfil axiológico solicitado. No contexto clínico, conhecer que valores regem com mais importância a vida de uma pessoa, auxilia o psicólogo a entender melhor seu paciente, seus anseios e suas metas ao longo da vida. Porém, é importante destacar que o QVB-D não substitui ou elimina o uso do QVB em suas formas mais reduzidas, como o que tem sido empregado em múltiplas pesquisas com 18 itens (Gouveia, 2013; Medeiros, 2011).

Por fim, seguramente pesquisas futuras deverão ser realizadas com o instrumento proposto. Um estudo imprescindível poderá ser testar sua adequação em diversos campos de atuação do psicólogo, avaliando, por exemplo, sua validade preditiva no ajuste de um candidato a determinado cargo ou as escolhas de um cliente diante de um conflito entre o trabalho e a família. Porém, com o fim de que venha a ser utilizado para triagem e diagnóstico, urge que seja realizada sua normatização para o contexto brasileiro. Entretanto, este empreendimento implicará em esforço maior do que há podido ser feito neste estudo, considerando amostras de todas as partes do país. Deste modo, é que se vislumbra no futuro, podendo ocupar a agenda desses pesquisadores.

\section{Referências}

Allport, G. W., Vernon, P. E., \& Lindzey, G. (1960). Study of value. Manual and test booklet. ( $3^{\text {rd }}$ ed.). Boston, MA: Houghton Mifllin.

Bardi, A., \& Schwartz, S. H. (2003). Values and behavior: Strength and structure of relations. Personality and Social Psychology Bulletin, 29, 1207-1220. doi:10.1177/0146167203254602

Braithwaite, V. A., \& Scott, W. A. (1991). Values. In J. P. Robinson, P. R. Shaver, \& L. S. Wrightsman (Eds.), Measures of personality and social psychological attitudes (pp. 661-753). New York: Academic Press.

Brown, T. A. (2006). Confirmatory factor analysis for applied research. New York: The Guilford Press.

Byrne, B. M. (2010). Structural equation modeling with Amos: Basic concepts, applications, and programmimg ( $2^{\text {nd }}$ ed.). New York: Routledge.

Caprara, G. V., Schwartz, S. H., Capanna, C., Vecchione, M., \& Barbaranelli, C. (2006). Personality and politics: Values, traits, and political choice. Political Psychology, 27, 1-28. doi:10.1111/j.1467-9221.2006.00447.x

Clark, L. A., \& Watson, D. (1995). Constructing validity: Basic issues in objective scale development. Psychological Assessment, 7, 309-319. doi:10.1037/1040-3590.7.3.309
Devos, T., Spini, D., \& Schwartz, S. H. (2002). Conflicts among human values and trust in institutions. British Journal of Social Psychology, 41, 481-494. doi:10.1348/014466602321149849

Fornell, C., \& Larcker, D. F. (1981). Evaluating structural equation models with unobservable variables and measurement error. Journal of Marketing Research, 18, 39-50.

Garson, G. D. (2003). PA 765 Statnotes: An online textbook. Retrieved from http://faculty.chass.ncsu.edu/garson/PA765/ statnote.htm

Gibbins, K., \& Walker, I. (1993). Multiple interpretations of the Rokeach Value Survey. Journal of Social Psychology, 133, 797-805. doi:10.1080/00224545.1993.9713941

Goodwin, R., Realo, A., Kwiatkowska, A., Kozlova, A., Nguyen, L. L. A., \& Nizharadze, G. (2002). Values and sexual behavior in Central and Eastern Europe. Journal of Health Psychology, 7, 45-56. doi:10.1177/1359105302007001651

Gorsuch, R. L. (1970). Rokeach's approach to value systems and social compassion. Review of Religious Research, 11, 139-143.

Gouveia, V. V. (1998). La naturaleza de los valores descriptores del individualismo y del colectivismo: una comparación intra e intercultural (Tese de doutorado não publicada, Universidade Complutense de Madri, Espanha).

Gouveia, V. V. (2003). A natureza motivacional dos valores humanos: Evidências acerca de uma nova tipologia. Estudos de Psicologia (Natal), 8, 431-443.

Gouveia, V. V. (2013). Teoria funcionalista dos valores humanos: Fundamentos, aplicações e perspectivas. São Paulo, SP: Casa do Psicólogo.

Gouveia, V. V., Milfont, T. L., \& Guerra, V. M. (2014). Functional theory of human values: Testing its content and structure hypotheses. Personality and Individual Differences, 60, 41-47. doi:10.1016/j.paid.2013.12.012

Gouveia, V. V., Milfont, T. L., Fischer, R., \& Coelho, J. A. P. M. (2009). Teoria Funcionalista dos Valores Humanos: Aplicações para organizações. Revista de Administração Mackenzie, 10, 34-59.

Gouveia, V. V., Milfont, T. L., Fischer, R., \& Santos, W. S. (2008). Teoria Funcionalista dos Valores Humanos. In M. L. M. Teixeira (Ed.), Valores humanos e gestão: Novas perspectivas (pp. 47-80). São Paulo, SP: Serviço Nacional de Aprendizagem Comercial.

Gouveia, V. V., Milfont, T. L., Soares, A. K. S., Andrade, P. R., \& Leite, I. L. (2011). Conhecendo os valores na infância: Evidências psicométricas de uma medida. Pisco, 42, 106-115.

Gouveia, V. V., Santos, W. S., Milfont, T. L., Fischer, R., Clemente, M., \& Espinosa, P. (2010). Teoría funcionalista de los valores humanos en España: comprobación de las hipótesis de contenido y estructura. Revista Interamericana de Psicología, 44, 203-214.

Groot, J. I. M., \& Steg, L. (2008). Value orientation explain beliefs related to environmental significant behavior: How to measure egoistic, altruistic, and biospheric value orientations. Environment and Behavior, 40, 330-354. doi:10.1177/0013916506297831

Hair, J., Jr., Anderson, R., Tatham, R., \& Black, W. (1998). Multivariate data analysis $\left(5^{\text {th }} \mathrm{ed}\right.$.) Upper Saddel River, NJ: Prentice Hall.

Harding, S., \& Phillips, D. (1986). Contrasting values in Western Europe: Unity, diversity and change. London: Macmillan.

Inglehart, R. (1977). The silent revolution: Changing values and political styles among Western publics. Princeton, NJ: Princeton University Press. 
Souza, L. E. C., Gouveia, V. V., Lima, T. J. S. \& Santos, W. S. (2015). Questionários dos Valores Básicos - Diagnóstico (QVB-D): Evidências de Validade de Construto.

Inglehart, R., Basañez, M., \& Moreno, A. (1998). Human values and beliefs: A cross-cultural sourcebook: Political, religious, sexual and economic norms in 43 societies. Michigan, IL: The University of Michigan Press.

Kelly, T. A. (1990). The role of values in psychotherapy: A critical review of process and outcome effects. Clinical Psychology Review, 10, 171-186. doi:10.1016/0272-7358(90)90056-G

Kluckhohn, C. K. M. (1951). Values and value orientations in the theory of action. In V. Parsons \& E. Shils (Eds.), Toward a general theory of action (pp. 388-433). Cambridge, MA: Harvard University Press.

Maio, G. R. (2010). Mental representations of social values. In M. P. Zanna (Ed.), Advances in experimental social psychology (Vol. 42, pp. 1-43). Burlington, VT: Academic Press.

Maio, G. R., Olson, J. M., Bernard, M. M., \& Luke, M. A. (2006). Ideologies, values, attitudes, and behavior. In J. DeLamater (Ed.), Handbook of social psychology (pp. 283-308). Madison, WI: Springer.

Maslow, A. H. (1954). Motivation and personality. New York: Harper \& Row.

Medeiros, E. D. (2011). Teoria funcionalista dos valores humanos: Testando sua adequação intra e interculturalmente (Tese de doutorado não publicada, Universidade Federal da Paraíba, João Pessoa, PB, Brasil).

Milfont, T. L., Sibley, C. G., \& Duckitt, J. (2010). Testing the moderating role of the components of norm activation on the relationship between values and environmental behaviour. Journal of Cross-Cultural Psychology, 41, 124-131. doi:10.1177/0022022109350506

Morris. C. W. (1956). Varietie of human value. Chicago, IL: University of Chicago Press.

Nunnally, J. C. (1991). Teoría psicométrica. México, DF: Trillas.

Pasquali, L. (2003). Psicometria: Teoria dos testes na Psicologia e na Educação. Petrópolis, RJ: Vozes.

Roccas, S., \& Sagiv, L. (2010). Personal values and behavior: Taking the cultural context into account. Social and Personality Psychology, 4, 30-41. doi:10.1111/j.17519004.2009.00234.x

Rokeach, M. (1968). Beliefs, attitudes and values. San Francisco, CA: Jossey-Bass.

Rokeach, M. (1973). The nature of human values. New York: Free Press.

Santos, W. S. (2008). Explicando comportamentos socialmente desviantes: Uma análise do compromisso convencional e afiliação social (Tese de doutorado não publicada, Universidade Federal da Paraíba, João Pessoa, PB, Brasil).

Schwartz, S. H. (1992). Universals in the content and structure of values: Theoretical advances and empirical tests in 20 countries. In M. Zanna (Ed.), Advances in experimental social psychology (Vol. 25, pp. 1-65). New York: Academic Press.

Schwartz, S. H. (2005). Validade e aplicabilidade da teoria de valores. In A. Tamayo \& J. B. Porto (Eds.), Valores e comportamentos nas organizações (pp. 56-95). Rio de Janeiro, RJ: Vozes.

Schwartz, S. H., \& Bilsky, W. (1987). Toward a universal psychological structure of human values. Journal of Personality and Social Psychology, 53, 550-562. doi:10.1037/00223514.58.5.878

Schwartz, S. H., \& Huismans, S. (1995). Value priorities and religiosity in four Western religions. Social Psychological Quarterly, 58, 88-107.
Smith, P. B., \& Schwartz, S. H. (1997). Values. In J. W. Berry, C. Kagitcibasi, \& M. H. Segall (Eds.), Handbook of crosscultural psychology (Vol. 3, pp. 77-119). Boston, MA: Allyn \& Bacon.

Tamayo, A. (2005). Impacto dos valores pessoais e organizacionais sobre o comprometimento organizacional. In A. Tamayo \& J. B. Porto (Eds.), Valores e comportamento nas organizações (pp. 160-186). Rio de Janeiro, RJ: Vozes.

Tamayo, A. (2007). Hierarquia de valores transculturais e brasileiros. Psicologia: Teoria e Pesquisa, 23, 7-15.

Tamayo, A., Lima, A., Marques, J., \& Martins, L. (2001). Prioridades axiológicas e uso de preservativo. Psicologia: Reflexão e Crítica, 14, 151-159.

Vasconcelos, T. C., Gouveia, V. V., Souza, M. L., Filho, Sousa, D. M., \& Jesus, G. R. (2004). Preconceito e intenção em manter contato social: Evidências acerca dos valores humanos. Psico-USF, 9, 147-154.
Recebido: $27 / 01 / 2014$

$1^{a}$ revisão: 25/04/2014

Aceite final: 15/05/2014 